\title{
Analysis of the effects of prepared porcelain veneers and unprepared porcelain veneers on gingival crevicular flora based on high-throughput sequencing
}

\author{
RUI ZHANG ${ }^{1}$, LANHUA SHEN ${ }^{1}$, DANDAN XU ${ }^{2,3}$ and XIAO LI ${ }^{2,3}$ \\ ${ }^{1}$ Department of Stomatology, Nangang Branch, Heilongjiang Provincial Hospital, Harbin, Heilongjiang 150000; \\ ${ }^{2}$ Department of Stomatology, The First Hospital of Qiqihar; ${ }^{3}$ Department of Stomatology, Affiliated Qiqihar Hospital, \\ Southern Medical University, Qiqihar, Heilongjiang 161006, P.R. China
}

Received June 18, 2020; Accepted February 23, 2021

DOI: 10.3892/etm.2021.10209

\begin{abstract}
The effect of tooth preparation on the gingival crevicular flora of abutment teeth during porcelain veneer treatment is not clear. The purpose of the present study was to analyze the difference between prepared porcelain veneers and unprepared porcelain veneers on gingival crevicular flora. High-throughput sequencing was used in the present study. A total of 20 patients (40 anterior teeth) with veneer restoration of anterior teeth were enrolled. They were divided into two groups: The prepared porcelain veneer group (group P, 11 cases, 19 anterior teeth) and unprepared porcelain veneer group (group U, 9 cases, 21 anterior teeth). After 2 years of follow-up, the restoration and healthy natural gingival crevicular fluid were collected to extract bacterial DNA. 16S ribosomal DNA high-throughput sequencing technique was used to compare the diversity of gingival sulcus flora structure between the prepared porcelain veneer and unprepared porcelain veneer groups. In addition, a healthy control group (group H) was also used for comparison. The Shannon index of the group $\mathrm{U}$ was lower than that of group $\mathrm{H}$ and group $\mathrm{P}$. The abundance of Proteus in group $U$ was higher than that in group $\mathrm{H}$ and group $\mathrm{P}$ at the phylum level $(\mathrm{P}<0.05)$. At the genus level, the abundance of Porphyromonas, Prevotella and Actinomycetes in group $U$ was significantly higher than that in group $\mathrm{H}$ and group $\mathrm{P}(\mathrm{P}<0.05)$. Compared with the group $\mathrm{P}$, the bacterial diversity of the group $U$ was lower, and the proportion of gingival sulcus pathogenic bacteria was higher. The unprepared porcelain veneer had a certain adverse effect on the periodontal tissue.
\end{abstract}

Correspondence to: Dr Xiao Li, Department of Stomatology, The First Hospital of Qiqihar, 30 Park Road, Longsha, Qiqihar, Heilongjiang 161006, P.R. China

E-mail: lixiaomaisui@163.com

Key words: high-throughput sequencing, gingival crevicular flora, porcelain veneers, tooth preparation

\section{Introduction}

With the development of dental cosmetic repair technology in recent years, the number of patients who visit dental clinics for dental cosmetic repair is increasing. The scope of repairs mainly includes anterior tooth defects and gaps, abnormal tooth color, distorted teeth or microdontia (1). Porcelain veneer repair method is increasingly recognized and requested by patients. During treatment, no tooth preparation is required, or only a small amount of cavitation has to be removed. The enamel structure can be protected. At present, due to its advantages of less injury, high biocompatibility and beauty, it plays an increasingly important role in minimally invasive tooth repair $(1,2)$. Currently, porcelain veneer restoration is divided into prepared porcelain veneers and unprepared porcelain veneers. In prepared porcelain veneers, the enamel needs to be partially ground, leaving enough thickness for the veneer to enhance its strength and color authenticity. Unprepared porcelain veneers do not require tooth preparation nor do they damage teeth (3). With the promotion of porcelain veneer therapy in clinics, periodontal health reflects the effect of porcelain veneer therapy. Gingival inflammation is one of the common complications of tooth restoration (4). Gingival crevicular fluid is an important medium for the growth of periodontal flora, and changes in gingival crevicular flora are closely related to periodontitis (5-8). Whether tooth preparation is required when using porcelain veneers and whether the veneers affect gingival crevicular flora are controversial. These factors are of great significance for the selection of veneer treatment methods.

At present, studies on gingival crevicular flora mainly focus on specific pathological states (such as periodontitis or some systemic diseases) (9). Relevant research has revealed that the composition of gingival crevicular flora has specificity compared with healthy states under conditions such as periodontitis and gingivitis (9). In some systemic diseases such as liver cirrhosis, systemic lupus erythematosus and IgA nephropathy, there is a correlation between the changes of subgingival microbial structure and the occurrence of diseases (10-12). Therefore, changes in the composition of gingival crevicular flora could reveal to some extent the health 
status of periodontal tissue. However, whether tooth preparation affects periodontal health through changes in gingival crevicular flora has not yet been reported. Compared with traditional bacterial culture methods, high-throughput sequencing technology has outstanding advantages such as high accuracy, high throughput, high sensitivity and low operating cost. It can quickly and accurately reflect the composition and diversity of microorganisms and has been widely used in the field of microbial research $(13,14)$. The aim of the present study was to analyze the influence of tooth preparation on the composition and diversity of gingival crevicular flora. High-throughput sequencing technique was used to determine the influence of prepared porcelain veneers and unprepared porcelain veneers on periodontal health.

\section{Patients and methods}

Research subjects. A total of 20 patients were selected as research subjects, with a total of 40 teeth. They received treatment with anterior dental veneers at Nangang Branch, Heilongjiang Provincial Hospital (Harbin, China) from January 2016 to December 2017. The group using unprepared porcelain veneers was considered as the observation group (group U). There were 9 patients (21 affected teeth in total) in this group, including 4 males ( 8 teeth), 5 females (13 teeth), 14 anterior teeth and 7 mandibular anterior teeth. The group using prepared porcelain veneers was considered as the control group (group P). There were 11 patients (19 affected teeth in total) in this group, including 5 males (10 teeth), 6 females (9 teeth), 12 anterior teeth and 7 mandibular anterior teeth. Twenty healthy natural teeth of healthy people were selected as healthy controls (group H). The patients were 18-44 years old, with an average age of $(28.36 \pm 5.39)$ years.

Inclusion criteria for the porcelain veneer patients were as follows: i) All the patients met the clinical criteria of porcelain veneer repair; ii) patients were in good mental state; iii) patients who had not used antibiotics, hormone drugs or received radiotherapy and chemotherapy in the past three months; and iv) patients who had no abnormal secretions in the mouth. Exclusion criteria were as follows: i) Patients complicated with dental pulp and periodontal inflammation, odontatrophy, or loosened teeth before surgery; ii) patients with contraindications to surgery; iii) patients unwilling to cooperate or who ground their teeth at night; iv) female pregnant or lactating patients; v) patients combined with known systemic diseases (AIDS, tuberculosis, hepatitis, known history of any other infectious diseases, diabetes, ischemic heart disease, hypertension, thyroid or other hormone disorders, autoimmune diseases and cancer); vi) patients with abnormal coagulation function; and vii) patients who failed to cooperate with the follow-up. There was no statistical difference in the general data between the two groups ( $\mathrm{P}>0.05)$ (Table I).

Inclusion criteria for group $\mathrm{H}$ were as follows: i) All the subjects were aged $18-65$ years; ii) subjects were in good mental state; iii) subjects who did not use antibiotics, hormone drugs or receive radiotherapy and chemotherapy in the past three months; and iv) subjects who had no abnormal secretions in the mouth. Exclusion criteria were as follows: i) Subjects complicated with dental pulp and periodontal inflammation, odontatrophy, or loosened teeth; ii) subjects who had received dental prosthesis treatment (including porcelain veneers, resin veneers and porcelain full crowns); iii) subjects unwilling to cooperate or who ground their teeth at night; iv) female pregnant or lactating subjects; v) subjects combined with known systemic diseases (AIDS, tuberculosis, hepatitis, known history of any other infectious diseases, diabetes, ischemic heart disease, hypertension, thyroid or other hormone disorders, autoimmune diseases and cancer); vi) subjects with abnormal coagulation function and vii) subjects who failed to cooperate with the follow-up.

The study was approved by the Ethics Committee of Nangang Branch of Heilongjiang Provincial Hospital and written informed consent was obtained from all patients.

Therapeutic method. The therapeutic methods were as follows: Group P, after color comparison and photo recording, tooth preparation, temporary restoration and veneering were carried out according to conventional procedures (14). The parameters were as follows: i) The thickness of tooth abrasion was $0.4-0.6 \mathrm{~mm}$; ii) dental prosthesis treatment was designed with butt-type cutting ends, with gingival margins; the neck margin was designed as an angular shoulder, and the width was controlled at $0.3-0.5 \mathrm{~mm}$; iii) the veneer material was IPSe and the Emax porcelain veneer was completed by the same producer (after 7 days, the patients were re-examined and if necessary, porcelain veneers could be adjusted or even re-made); iv) the tooth surface was treated with $37 \%$ phosphoric acid for $30 \mathrm{sec}$ and the porcelain veneer was treated with hydrofluoric acid for $15 \mathrm{sec}$; and v) excess adhesive was carefully removed and polished when necessary. Group U, tooth preparation and temporary restoration were not required. Veneer design requirements were the same as those of group $\mathrm{P}$, except that the phosphoric acid treatment time of the tooth surface was extended to $60 \mathrm{sec}$.

Both groups of patients were provided with oral health care guidance after treatment.

Patient follow-up. Follow-up visits were conducted in the 1st, 3rd, 6th, 12th and 24th month after surgery. The gingival health and the success rate of restorations were evaluated based on the clinical evaluation criteria of the California Dental Association (15) and the modified Ryge evaluation criteria (16). The success rate of restoration was calculated 2 years later.

Gingival health evaluation criteria $(12,13)$ were as follows: i) Healthy gingiva; ii) slight gingival inflammation and a small amount of bleeding could be detected, and slight gingival atrophy that did not affect the appearance could be observed; and iii) gingival swelling was obvious, bleeding and periodontal pockets deepened, accompanied by moderate to severe gingival atrophy, affecting the appearance.

Evaluation criteria for successful restoration (14) were as follows: The size and shape of the restored tooth were coordinated with the adjacent teeth and antagonist teeth; the color was consistent, no discoloration occurred; the teeth were in good occlusion, and there was no gap between the teeth under naked eye observation; the occlusal performance of the teeth was good, and there was no visible gap between the teeth; there were no symptoms of swollen gums and sore teeth.

Sample collection. Samples were obtained 2 years after restoration from the affected tooth and healthy natural gingival sulcus near the buccal profile. Subjects were advised to avoid 
Table I. Basic characteristics of patients.

\begin{tabular}{lccc}
\hline Characteristics & U group $(\mathrm{n}=21)$ & P group $(\mathrm{n}=19)$ & P-value \\
\hline Sex (male:female) & $8(38.1 \%): 13(61.9 \%)$ & $10(52.6 \%): 9(47.4 \%)$ & 0.163 \\
Age (years) & $30.27 \pm 5.33$ & $27.64 \pm 3.19$ & \\
Blood results & & & \\
WBC & $6.02 \pm 2.03$ & $5.91 \pm 1.56$ & 0.132 \\
CRP & $1.19 \pm 0.40$ & $1.96 \pm 1.33$ & 0.151 \\
ALT & $10.40 \pm 3.90$ & $12.92 \pm 8.43$ & 0.560 \\
AST & $15.30 \pm 11.98,19.60)$ & $16.91 \pm 12.83$ & 0.118 \\
BUN & $3.64 \pm 1.92$ & $3.21 \pm 1.97$ & 0.106 \\
Scr & $94.15 \pm 16.35$ & $96.55 \pm 14.51$ & 0.474 \\
\hline
\end{tabular}

P, prepared porcelain veneers; $\mathrm{U}$, unprepared porcelain veneers.

oral cleaning such as tooth brushing and toothwash on the sampling day. Subjects were also requested to gargle with sterile water $20 \mathrm{~min}$ before sampling to remove the stained supragingival plaque around the sampling area. Sterile cotton balls were used for isolation. The sterile test paper of the same specification was placed in the gingival crevicular for $30 \mathrm{sec}$. After the gingival crevicular fluid was acquired, it was quickly placed into a sterile transport tube, marked, and stored in a $-80^{\circ} \mathrm{C}$ freezer. Samples contaminated by blood stains and saliva were excluded during sampling.

\section{Operating methods}

Bacterial $16 S$ rDNA extraction, PCR amplification and sequencing. According to the manufacturer's instructions, the total DNA of bacteria was extracted using PowerSoil ${ }^{\circledR}$ DNA isolation kit (cat. no. 12888-100; Mo Bio Laboratories, Inc.). The total DNA content was determined using Nanodrop ND-2000 (Thermo Fisher Scientific, Inc.). PCR amplification was applied in the 16S rDNA V3-V4 gene hypervariable region. The universal primer sequences were 357F (5'-CCTACGGGAGGCAGC AG-3') and 806R (5'-GGACTACHVGGGTWTCTAAT-3'). PCR was carried out on a MasterCycle gradient (Eppendorf). The reaction system was $50 \mu \mathrm{l}$ : Including $5 \mu \mathrm{l}$ of $10 \mathrm{X}$ Ex-Taq buffer (Mg2+Plus) (Takara Biotechnology Co., Ltd.), $4 \mu 1$ of dNTP mixture (12.5 mM each) (Takara Biotechnology Co., Ltd.), 1.25 units Ex-Taq DNA Polymerase (Takara Biotechnology Co., Ltd.), $2 \mu 1$ Template DNA (Allwegene Tech.), $200 \mathrm{nM}$ upstream primers and $200 \mathrm{nM}$ downstream primers (Allwegene Tech.) and $36.75 \mu \mathrm{l}$ double-distilled water (Takara Biotechnology Co., Ltd.). PCR reaction conditions were as follows: Pre-denaturation at $94^{\circ} \mathrm{C}$ for $2 \mathrm{~min} ; 94^{\circ} \mathrm{C}$ for $30 \mathrm{sec}, 57^{\circ} \mathrm{C}$ for $30 \mathrm{sec}, 72^{\circ} \mathrm{C}$ for $30 \mathrm{sec}$, with a total of $30 \mathrm{cycles}$, and finally, extension at $72^{\circ} \mathrm{C}$ for $10 \mathrm{~min}$. Three PCR products were collected from each sample to reduce reaction-level PCR bias. The PCR product was purified by QIAquick Gel Extraction kit (Qiagen $\mathrm{GmBH}$ ) and quantified by real-time PCR. High-throughput sequencing (5) was carried out at Allwegene Tech. The sequencing platform was Illumina HiSeq 2500 (Illumina, Inc.).

Bioinformatics analysis. Species annotation and dilution curves were based on operational taxonomic unit (OTU) representative sequences using v.1.13.0 Mothur software (6) and GreenGene database (threshold: 0.8-1.0) (7). QIIME software (v1.9.1) was applied to calculate the Alpha diversity value (Shannon index) of a single sample (8).

Statistical analysis. All data were expressed as the mean \pm standard deviation. Independent sample t-test was used to compare the Alpha diversity among groups. Wilcoxon rank sum test was used to compare the level of flora among groups. Veneer retention rate and periodontal health were detected by chi-square test using SPSS 22.0 software (IBM Corp.). $\mathrm{P}<0.05$ was considered to indicate a statistically significant difference.

\section{Results}

Evaluation of gingival health at different time-points of reexamination. Evaluation of gingival health during the follow-up visit of groups $\mathrm{P}$ and $\mathrm{U}$ is presented in Table II. Within 2 years, slight gingival inflammation occurred in 4 cases of patients with unprepared porcelain veneers and 1 case of patients with prepared porcelain veneers and the difference was not statistically significant $(\mathrm{P}=0.342)$.

Comparison of the success rate of the two groups. The success rates of groups $\mathrm{P}$ and $\mathrm{U}$ are presented in Table III. The results revealed that there was no significant difference between the two groups.

Validity of sequencing results. In the present study, a total of 60 gingival crevicular fluid samples were obtained from 30 subjects. They were divided into 3 groups: The healthy group (group $\mathrm{H}, \mathrm{n}=20$ ), the unprepared porcelain veneer group (group $\mathrm{U}, \mathrm{n}=21$ ) and the prepared veneer group (group $\mathrm{P}, \mathrm{n}=19$ ). The original data were analyzed. After quality evaluation and screening, the effective data accounted for more than $98 \%$. The sequence length was concentrated at 450-600 bp, and the average length was longer than $500 \mathrm{bp}$, which met the final data analysis requirements (Fig. 1).

At this sequencing depth, the rarefaction curves of the three groups of samples (Fig. 2) gradually leveled off with the increase of ordinal numbers. It indicated that the sequencing depth had basically covered all species in the samples. The 
Table II. Evaluation of gingival health at different time-points of reexamination.

\begin{tabular}{|c|c|c|c|c|c|c|c|c|}
\hline Groups & Gum health level $^{\mathrm{a}}$ & 1 st month & 3rd month & 6th month & 12th month & 24th month & Total adverse reactions & $\mathrm{P}$-value \\
\hline \multirow[t]{3}{*}{$\mathrm{U}(\mathrm{n} / \%)$} & A & $20(100)$ & $20(100)$ & $19(95)$ & $18(90)$ & $17(85)$ & $4(20)$ & 0.342 \\
\hline & B & 0 & 0 & $1(5)$ & $2(5)$ & $1(5)$ & & \\
\hline & $\mathrm{C}$ & 0 & 0 & 0 & 0 & 0 & & \\
\hline \multirow[t]{3}{*}{$\mathrm{P}(\mathrm{n} / \%)$} & A & $20(100)$ & $20(100)$ & $20(100)$ & $19(95)$ & $19(95)$ & $1(5)$ & \\
\hline & B & 0 & 0 & 0 & $1(95)$ & 0 & & \\
\hline & $\mathrm{C}$ & 0 & 0 & 0 & 0 & 0 & & \\
\hline
\end{tabular}

${ }^{a} \mathrm{~A}$, healthy gums; B, mild gum inflammation, a small amount of bleeding could be detected, mild atrophy of the gums that did not affect the appearance could be observed; $\mathrm{C}$, gums had obvious redness and swelling, bleeding, as well as deepened periodontal pockets, accompanied by moderate to severe gum shrinkage which affected appearance. U, unprepared porcelain veneers; P, prepared porcelain veneers.

Table III. Comparison of the success rates of groups $\mathrm{P}$ and $\mathrm{U}$ after 2 years.

\begin{tabular}{lcccc}
\hline Groups & Successful (n) & Failed (n) & Total (n) & Success rate (\%) \\
\hline $\mathrm{P}$ & 17 & 3 & 20 & 85 \\
$\mathrm{U}$ & 19 & 1 & 30 & 95 \\
\hline
\end{tabular}

P, prepared porcelain veneers; $\mathrm{U}$, unprepared porcelain veneers.

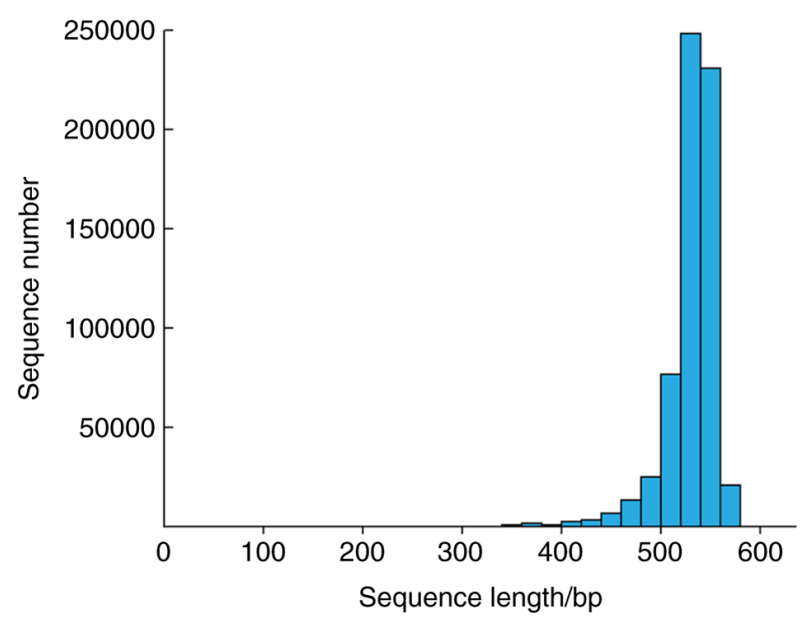

Figure 1. Length of distribution of raw reads. In total, 30 groups of original data were sequenced; the sequence length was concentrated at 450-600 bp, and the average length was $>500 \mathrm{bp}$.

sampling and sequencing results could basically reflect the real microbial community status of the obtained samples.

Analysis of flora diversity in samples from dental restoration two years later. There was a significant difference in the Alpha diversity index among the 3 groups of samples $(\mathrm{P}<0.05)$. Group $\mathrm{H}$ had the highest Shannon index, followed by group $\mathrm{U}$, and group $\mathrm{P}$ had the lowest index, as revealed in Fig. 3.

Difference analysis on composition of bacterial flora at phylum level among samples. A total of 12 bacterial phyla were obtained from all samples. The top five dominant

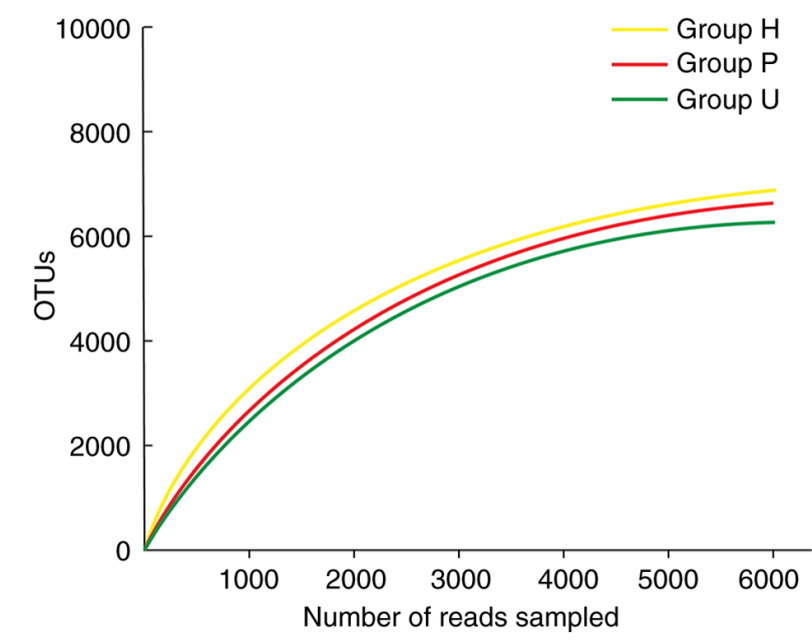

Figure 2. Rarefaction curves of three groups. With the increase of sequencing number of the three groups of samples, the dilution curve gradually increased. When the number of sequencing exceeded 3000, the dilution curve reached the plateau stage, indicating that the sequencing depth had basically covered all species in the sample, and the sampling and sequencing results could reflect the real microbial community status of the obtained samples. OTUs, operational taxonomic units; $\mathrm{H}$, healthy control; $\mathrm{P}$, prepared porcelain veneers; U, unprepared porcelain veneers.

phylum with higher abundance at the phylum level were Firmicutes, Bacteroidetes, Proteobacteria, Fusobacteria and Actinobacteria, as revealed in Fig. 4. The abundance of dominant bacteria in each group was basically the same, however, the difference of Proteobacteria in the three groups was statistically significant $(\mathrm{P}<0.01)$. The proportion of Proteobacteria in gingival crevicular fluid in group $U$ was higher than that in group $\mathrm{P}$ and group $\mathrm{H}$. 


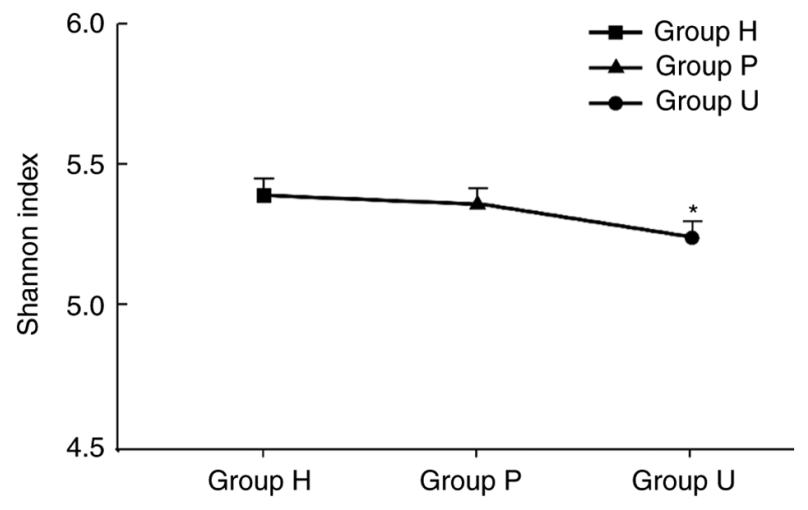

Figure 3. Shannon index difference between groups. The Shannon indexes of groups $\mathrm{H}, \mathrm{P}$ and $\mathrm{U}$ were 5.41, 5.38 and 5.22, respectively, and the difference between groups was statistically significant $\left({ }^{\prime} \mathrm{P}<0.05\right)$. This indicated that the species diversity of the periodontal microecological environment in group $\mathrm{U}$ was decreased, suggesting that periodontal tissue after treatment with unprepared porcelain veneers may be in a certain pathological stage due to the pathogen aggregation and formation of a core pathogenic complex. $\mathrm{H}$, healthy control; P, prepared porcelain veneers; $\mathrm{U}$, unprepared porcelain veneers.

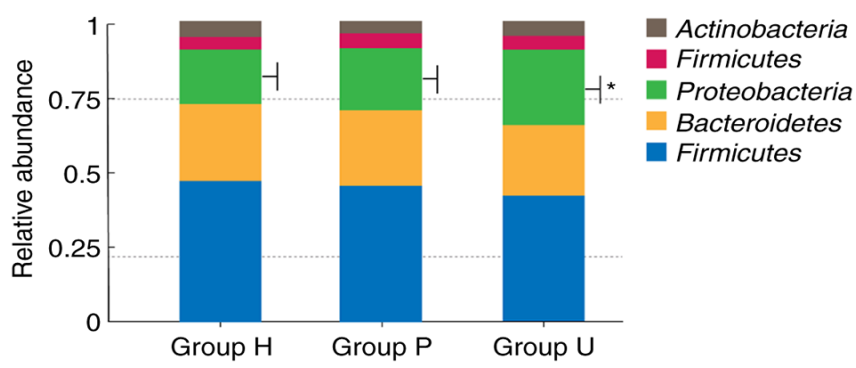

Figure 4. Relative abundance of species in oral microbial community of each group at the phylum level. This chart revealed the first five dominant phyla with high abundance in each group at the phylum level, which were Firmicutes, Bacteroidetes, Proteobacteria, Fusobacteria and Actinobacteria. The abundance of dominant bacteria in each group was basically the same, but the difference of Proteobacteria in these three groups was statistically significant $(" \mathrm{P}<0.01)$. The proportion of Proteobacteria in gingival crevicular fluid of group $\mathrm{U}$ was higher than that of group $\mathrm{P}$ and group $\mathrm{H}$. $\mathrm{H}$, healthy control; $\mathrm{P}$, prepared porcelain veneers; $\mathrm{U}$, unprepared porcelain veneers.

Difference analysis of flora composition at genus level among samples. The top 10 genera with the highest relative abundance were Streptococcus, Neisseria, Prevotella-7, Prevotella, Actinobacillus, Veillonella, Porphyromonas, Prevotella-6, Gemella and Leptotrichia. Among them, Porphyromonas, Prevotella and Actinobacillus in gingival crevicular fluid of group U had statistically significant differences compared with those in group $\mathrm{P}$ and group $\mathrm{H}(\mathrm{P}<0.01)$. The distribution is presented in Fig. 5.

\section{Discussion}

Anterior teeth influence the appearance of the face to a great extent. In the past, resin veneers and porcelain full crowns were the main methods to repair the anterior teeth. To provide suitable space for the restoration in order for the denture and the abutment to have good retention and close contact, the full crown restoration required grinding off the original tooth enamel layer in advance. However, the color of the resin veneers was unstable and the molar quantity of

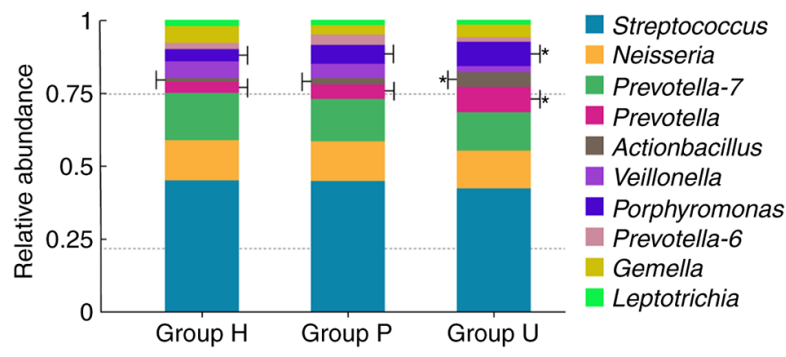

Figure 5. Relative abundance of species in oral microbial community of each group at genus level. The top 10 genera with the largest relative abundance were Streptococcus, Neisseria, Prevotella-7, Prevotella, Actinobacillus, Veillonella Porphyromonas, Prevotella-6, Gemella and Leptotrichia. Porphyromonas, Prevotella and Actinobacillus in gingival crevicular fluid of group U were significantly different from those in group $\mathrm{P}$ and group $\mathrm{H}\left({ }^{*} \mathrm{P}<0.01\right) . \mathrm{H}$, healthy control; $\mathrm{P}$, prepared porcelain veneers; $\mathrm{U}$, unprepared porcelain veneers.

the porcelain full crowns was excessive, which limited these two methods in clinical practice (17). The advent of porcelain veneer technology has broken through this bottleneck. Due to its characteristics including minimally invasive and less enamel damage, the stability of restoration has increased, which greatly improves the clinical success rate (18). Porcelain materials have great biocompatibility, and concurrently, they can maintain favorable teeth aesthetics and patient satisfaction (19). Numerous studies, with evaluation time-points ranging from 5 to 20 years, support that porcelain veneer therapy exhibits favorable clinical performance (20-24). However, porcelain veneer treatment still has complications of varying degrees. The incidence of postoperative sensitivity is $>20 \%$, and the incidence of postoperative pulpitis is approximately $2.1 \%(25,26)$. Porcelain veneer treatment can be divided into prepared treatment and unprepared treatment. At present, relevant studies have revealed that there is no significant difference in periodontal adverse reactions and complication rates with or without dental preparation (18). This is consistent with the results of the present study.

Gingival crevicular flora is closely related to periodontal tissue health. The species and quantity composition ratio of gingival crevicular flora affect the balance of the periodontal micro-ecosystem (27). The samples of gingival crevicular flora were selected from patients after two years of treatment for high-throughput sequencing, and the microbial community mechanism of gingival crevicular flora of patients with and without tooth preparation was compared.

Alpha diversity can reflect species richness in the region, and Shannon index is positively correlated with the proportion of species diversity in the whole (28). Some studies $(29,30)$ have revealed that species diversity in the microecological environment would be reduced due to the accumulation of pathogenic bacteria. Then core pathogenic complexes under certain disease states would be formed. The results of the present study revealed that the Shannon index of group U was the lowest among the three groups, and the difference among the groups was statistically significant $(\mathrm{P}<0.05)$. It suggested that periodontal tissue may be in a certain pathological stage after the treatment of unprepared porcelain veneer.

Proteobacteria is the main species composition of gingival crevicular flora, which would increase under pathological conditions. It has been demonstrated to be one of the 
pathogenic bacteria of periodontal inflammation in dental implants $(30,31)$. There was a significant difference in the phylum level of Proteobacteria in gingival crevicular flora among the three groups, and the relative abundance in group $\mathrm{U}$ was higher than that in group $\mathrm{H}$ and group $\mathrm{P}$, with a significant difference. Porphyromonas, Prevotella and Actinobacillus are closely related to the onset of the disease (26). in the present, the abundance of these three types of bacteria in gingival crevicular fluid of group $\mathrm{U}$ was higher than that of group $\mathrm{P}$ and group $\mathrm{H}$, and the difference was statistically significant. These results suggest that unprepared porcelain veneers may increase the number of pathogenic bacteria in gingival crevicular fluid, which has certain adverse effects on periodontal health. The position and protrusion of the gingival margin of porcelain veneers are important factors for the influence of porcelain veneer treatment of periodontal tissue. The subgingival margin is considered to have a negative impact on periodontal health (29). A previous study suggests that the subgingival margin can change the normal protrusion of the root surface due to the marginal shape protrusion of the prosthesis, affecting the periodontal tissue (32). Therefore, in order to prevent the subgingival edge from overstimulating periodontal tissue, the gingival-aligned edge was used in the present study. Due to the lack of tooth preparation, a thin veneer edge may form certain overhang, stimulate gums and affect periodontal tissue. It can be observed from Table II that in the long-term follow-up, individual patients had minor adverse reactions such as minor periodontal bleeding, but no more serious periodontal complications such as grade $\mathrm{C}$ and above, indicating that the long-term prognosis of the patients was favorable. One reason may be that unprepared porcelain veneer treatment is minimally invasive, which minimizes the damage to the original periodontal microenvironment due to the non-grinding of tooth tissue. Although the periodontal flora may be in an unbalanced state due to the existence of overhang, its fluctuation range was small. There was no obvious pathological state from the macro perspective. However, the follow-up time of this study was short, and whether the current flora distribution led to long-term lesions remains unclear.

In previous studies, patients with unprepared porcelain veneers had a higher success rate of restoration and quality of life compared to those with prepared porcelain veneers $(33,34)$, however, some clinicians also maintain that unprepared porcelain veneers would lead to oversize of teeth and impair oral esthetics. In addition, they can easily breed bacteria, adversely affecting periodontal tissue (35-38). The present study also revealed that the unprepared porcelain veneers had a greater adverse impact on periodontal tissue in terms of micro-biology, and had potential risks to a certain extent. At present, tooth preparation often exceeds enamel, causing postoperative sensitivity, thus affecting the long-term curative effect. However, with the popularity of the concept of precision medicine, personalized treatment programs could meet the needs of patients to a greater extent. The development of micro-minimally invasive technology and the progress of related material technology have brought porcelain veneer treatment a broader prospect and more selections.

The duration of the present study was 2 years, and the number of cases involved was relatively small, which were the limitations of the this study. The effect of prepared porcelain veneers and unprepared porcelain veneers on gingival crevicular microflora can only be analyzed to a certain extent in the short term. A larger sample size and a longer study duration are required to guide the proper clinical application of tooth preparation.

\section{Acknowledgements}

Not applicable.

\section{Funding}

This work was supported by Heilongjiang Provincial Health Commission (2017-492).

\section{Availability of data and materials}

The datasets used and/or analyzed during the present study are available from the corresponding author on reasonable request.

\section{Authors' contributions}

RZ and XL conceived and designed this study. LS offered administrative support. All the authors prepared the materials for study. LS, DX and XL helped with data collection and summary. RZ and XL were responsible for data analysis and interpretation. $\mathrm{RZ}$ wrote the manuscript. All the authors read and approved the final manuscript.

\section{Ethics approval and consent to participate}

The study was approved by the Ethics Committee of Nangang Branch of Heilongjiang Province Hospital. Signed written informed consents were obtained from the patients.

\section{Patient consent for publication}

Not applicable.

\section{Competing interests}

The authors declare that they have no competing interests.

\section{References}

1. Park DJ, Yang JH, Lee JB, Kim SH and Han JS: Esthetic improvement in the patient with one missing maxillary central incisor restored with porcelain laminate veneers. J Adv Prosthodont 2: 77-80, 2010.

2. Horvath S and Dent DM: Minimally invasive restoration of a maxillary central incisor with a partial veneer. Eur J Esthet Dent 7: 6-16, 2012.

3. Zhang H, Sun Y, Guo J, Meng M, He L, Tay FR and Zhang S: The effect of food medium on the wear behaviour of veneering porcelain: An in vitro study using the three-body abrasion mode. J Dent 83: 87-94, 2019.

4. Mombelli A, Müller N and Cionca N: The epidemiology of peri-implantitis. Clin Oral Implants Res 23 (Suppl 6): S67-S76, 2012.

5. Khan AS, Ng SHS, Vandeputte O, Aljanahi A, Deyati A, Cassart JP, Charlebois RL and Taliaferro LP: A multicenter study to evaluate the performance of high-throughput sequencing for virus detection. $\mathrm{mSphere} 2$ : e00307-e00317, 2017.

6. Coelho PG, Bonfante EA, Silva NR, Rekow ED and Thompson VP: Laboratory simulation of Y-TZP all-ceramic crown clinical failures. J Dent Res 88: 382-386, 2009. 
7. Eraslan O, Aykent F, Yücel MT and Akman S: The finite element analysis of the effect of ferrule height on stress distribution at post-and-core-restored all-ceramic anterior crowns. Clin Oral Investig 13: 223-227, 2009.

8. Baek K, Ji S and Choi Y: Complex intratissue microbiota forms biofilms in periodontal lesions. J Dent Res 97: 192-200, 2018

9. Carda-Diéguez M, Bravo-González LA, Morata IM, Vicente A and Mira A: High-throughput DNA sequencing of microbiota at interproximal sites. J Oral Microbiol 12: 1687397, 2019.

10. Cao Y, Qiao M, Tian Z, Yu Y, Xu B, Lao W, Ma X and Li W: Comparative analyses of subgingival microbiome in chronic periodontitis patients with and without IgA nephropathy by high throughput 16S rRNA sequencing. Cell Physiol Biochem 47: 774-783, 2018

11. Jensen A, Ladegaard Grønkjær L, Holmstrup P, Vilstrup H and Kilian M: Unique subgingival microbiota associated with periodontitis in cirrhosis patients. Sci Rep 8: 10718, 2018

12. Corrêa JD, Calderaro DC, Ferreira GA, Mendonça SM, Fernandes GR, Xiao E, Teixeira AL, Leys EJ, Graves DT and Silva TA: Subgingival microbiota dysbiosis in systemic lupus erythematosus: Association with periodontal status. Microbiome 5: 34, 2017.

13. Shin YH, Lee YN, Lee HH, Dong JK and Oh SC: Effect of application of ZirLiner ${ }^{\circledR}$ and blasting treatments on shear bond strength of zirconia-veneered porcelain interface. J Dent Rehabil Appl Sci 24: 113-127, 2008.

14. Deng B, Liu HC, Yi YF, Wang C, Wen N and Tian JM: Effects of veneering porcelain type on bending strength of dental Y-TZP/porcelain bilayered structure. Adv Mater Res 105-106: 524-527, 2010

15. Erdemir EO, Duran I and Haliloglu S: Effects of smoking on clinical parameters and the gingival crevicular fluid levels of IL-6 and TNF-alpha in patients with chronic periodontitis. J Clin Periodontol 31: 99-104, 2010

16. Kim DM, Koszeghy KL, Badovinac RL, Kawai T, Hosokawa I Howell TH and Karimbux NY: The effect of aspirin on gingival crevicular fluid levels of inflammatory and anti-inflammatory mediators in patients with gingivitis. J Periodontol 78: 1620-1626, 2007.

17. Son J, Tze WTY and Gardner DJ: Thermal behavior of hydroxymethylated resorcinol (HMR)-treated maple veneer. Wood Fiber Sci 37: 220-231, 2005.

18. De Vasconcellos DK, Özcan M, Maziero Volpato CÂ, Bottino MA and Yener ES: Strain gauge analysis of the effect of porcelain firing simulation on the prosthetic misfit of implant-supported frameworks. Implant Dent 21: 225-229, 2012.

19. Singh S, Sharma $P$ and Kumar M: Evaluation of the effects of $0.05 \%$ sodium hypochlorite and $0.12 \%$ chlorhexidine gluconate twice daily rinse on periodontal parameters and gingival crevicular fluid HSV1 and CMV levels in patients with chronic periodontitis: A multicentric study. Med J Armed Forces India 2: 102-109, 2020

20. Chai J, McGivney GP, Munoz CA and Rubenstein JE: A multicenter longitudinal clinical trial of a new system for restorations. J Prosthet Dent 77: 1-11, 1997.

21. Gemlamaz D and Ergin S: Clinical evalution of all-ceramic crowns. J Prosthet Dent 89: 189-196, 2002.

22. Ryge G: Clinical criteria. Int Dent J 30: 347-358, 1980.
23. Kemp PF and Aller JY: Bacterial diversity in aquatic and other environments: What 16S rDNA libraries can tell us. FEMS Microbiol Ecol 47: 161-177, 2004.

24. Peumans M, Van Meerbeek B, Lambrechts P and Vanherle G: Porcelain veneers: A review of the literature. J Dent 28: 163-177, 2000.

25. Aristidis GA and Dimitra B: Five-year clinical performance of porcelain laminate veneers. Quintessence Int 33: 185-189, 2002.

26. D'Arcangelo C, De Angelis F, Vadini M and D'Amario M: Clinical evaluation on porcelain laminate veneers bonded with light-cured composite: Results up to 7 years. Clin Oral Investig 16: 1071-1079, 2012.

27. Dumfahrt H and Schaffer H: Porcelain laminate veneers. A retrospective evaluation after 1 to 10 years of service: Part II clinical results. Int J Prosthodont 13: 9-18, 2000.

28. Peumans M, De Munck J, Fieuws S, Lambrechts P, Vanherle G and Van Meerbeek B: A prospective ten-year clinical trial of porcelain veneers. J Adhes Dent 6: 65-76, 2004.

29. Beier US, Kapferer I, Burtscher D and Dumfahrt H: Clinical performance of porcelain laminate veneers for up to 20 years. Int J Prosthodont 25: 79-85, 2012.

30. Chen JH, Shi CX, Wang M, Zhao SJ and Wang H: Clinical evaluation of 546 tetracycline-stained teeth treated with Cerinate laminate veneers. Zhonghua Kou Qiang Yi Xue Za Zhi 38 119-202, 2003 (In Chinese)

31. Costa FO, Ferreira SD, Cortelli JR, Lima RPE, Cortelli SC and Cota LOM: Microbiological profile associated with peri-implant diseases in individuals with and without preventive maintenance therapy: A 5-year follow-up. Clin Oral Investig 23: 3161-3171, 2019.

32. Koyanagi T, Sakamoto M, Takeuchi Y, Maruyama N, Ohkuma M and Izumi Y: Comprehensive microbiological findings in peri-implantitis and periodontitis. J Clin Periodontol 40: 218-226, 2013.

33. Allen B, Kon M and Bar-Yam Y: A new phylogenetic diversity measure generalizing the Shannon index and its application to phyllostomid bats. Am Nat 174: 236-243, 2009.

34. Galloway-Peña JR, Smith DP, Sahasrabhojane P, Ajami NJ, Wadsworth WD, Daver NG, Chemaly RF, Marsh L, Ghantoji SS, Pemmaraju $\mathrm{N}$, et al: The role of the gastrointestinal microbiome in infectious complications during induction chemotherapy for acute myeloid leukemia. Cancer 122: 2186-2196, 2016.

35. Tsubota K: Ten-year clinical observation of a porcelain laminate veneer seated with biological tissue adaptation (BTA) technique. J Oral Sci 59: 311-314, 2017.

36. Marsh PD: Microbial ecology of dental plaque and its significance in health and disease. Adv Dent Res 8: 263-271, 1994.

37. Reeves WG: Restorative margin placement and periodontium health. J Prosthet Dent 66: 733-736, 1991.

38. Kawai K, Urano M and Ebisu S: Effect of surface roughness of porcelain on adhesion of baeteria and their synthesizing glueans. J Prosthet Dent 83: 664-667, 2000. International (CC BY-NC-ND 4.0) License. 